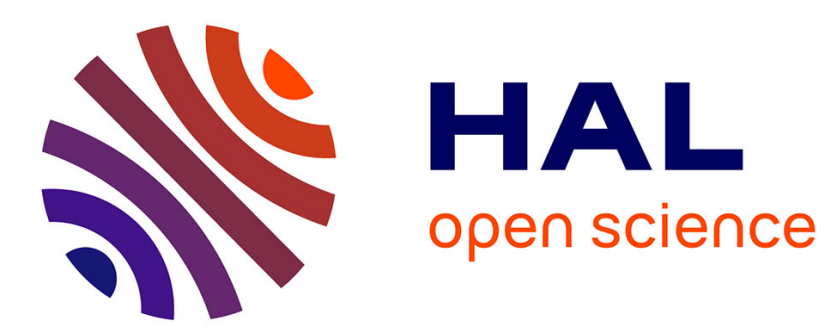

\title{
Robust finite-time output feedback stabilization of the double integrator
}

\author{
Emmanuel Bernuau, Wilfrid Perruquetti, Denis Efimov, Emmanuel Moulay
}

\section{To cite this version:}

Emmanuel Bernuau, Wilfrid Perruquetti, Denis Efimov, Emmanuel Moulay. Robust finite-time output feedback stabilization of the double integrator. International Journal of Control, 2014, 88 (3), pp.451460. 10.1080/00207179.2014.956340 . hal-01056207

\section{HAL Id: hal-01056207 https://hal.inria.fr/hal-01056207}

Submitted on 18 Aug 2014

HAL is a multi-disciplinary open access archive for the deposit and dissemination of scientific research documents, whether they are published or not. The documents may come from teaching and research institutions in France or abroad, or from public or private research centers.
L'archive ouverte pluridisciplinaire HAL, est destinée au dépôt et à la diffusion de documents scientifiques de niveau recherche, publiés ou non, émanant des établissements d'enseignement et de recherche français ou étrangers, des laboratoires publics ou privés. 


\title{
Robust finite-time output feedback stabilization of the double integrator
}

\author{
Emmanuel Bernuau, Wilfrid Perruquetti, Denis Efimov and Emmanuel Moulay
}

\begin{abstract}
The problem of finite-time output stabilization of the double integrator is addressed applying the homogeneity approach. A homogeneous controller and a homogeneous observer are designed (for different degrees of homogeneity) ensuring the finite-time stabilization. Their combination under mild conditions is shown to stay homogeneous and finite-time stable as well. Robustness and effects of discretization on the obtained closed loop system are analyzed. The efficiency of the obtained solution is demonstrated in computer simulations.
\end{abstract}

I

$\mathrm{N}$ many applications the nominal models have the double integrator form (mechanical planar systems, for instance). Despite its simplicity, this model is rather important in the control theory since frequently a design method developed for the double integrator can be extended to a more general case (via backstepping, for example). Most of the current techniques for nonlinear feedback stabilization provide an asymptotic stability: the obtained closed-loop dynamics is locally Lipschitz and the system trajectories settle at the origin when the time is approaching infinity. Such a rate of convergence is not admissible in many applications, this is why the Finite-Time Stability (FTS) notion is quickly developing during the last decades: solutions of a FTS system reach the equilibrium point in a finite time. For example, for $x \in \mathbb{R}$ and $\alpha \in(0,1)$, the solutions of $\dot{x}=-\operatorname{sign}(x)|x|^{\alpha}$ starting from $x_{0} \in \mathbb{R}$ at $t_{0}=0$ are

$$
\left\{\begin{array}{ll}
\operatorname{sign}\left(x_{0}\right)\left[\left|x_{0}\right|^{1-\alpha}-(1-\alpha) t\right]^{\frac{1}{1-\alpha}} & \text { if } \quad 0 \leq t \leq \frac{\left|x_{0}\right|^{1-\alpha}}{1-\alpha} \\
0 & \text { if } t>\frac{\left|x_{0}\right|^{1-\alpha}}{1-\alpha}
\end{array} .\right.
$$

Let us note that the right hand side of the above differential equation is not Lipschitz. In fact, finite-time convergence implies nonuniqueness of solutions (in backward time) which is not possible in the presence of Lipschitz-continuous dynamics, where different maximal trajectories never cross.

Engineers are interested in the FTS because one can manage the time for solutions to reach the equilibrium which is called the settling time. An important issue is the settling time function regularity at the origin, studied in [1] under the assumption of uniqueness of solutions in forward time. The problem of finite-time stability has been developed for continuous systems giving sufficient and necessary condition (see [2], [3]). In addition, necessary and sufficient conditions appear for discontinuous systems (see [4]). It was observed in many papers that FTS can be achieved if the system is locally asymptotically stable and homogeneous with negative degree [5]. This is why the homogeneity plays a central role in the FTS system design. The reader may found additional properties and results on homogeneity in [6], [7], [8], [9], [10]. The homogeneity property was used many times to design FTS state controls [11], [12], [13], [14], [15], [16], FTS observers [17], [18], [19], consensus protocols [20] and FTS output feedback [21], [22]. Particular attention was paid to triangular systems [23], [24].

The goal of the present work is twofold. First, a technique to design a FTS output feedback controller for the double integrator is presented. Since the double integrator is controllable, open-loop control strategies can be used to drive the state to the origin in a finite time (see [25], [26], [27] for a minimum time optimal control). Based on homogeneity, Bhat and Bernstein in their paper [11] provided a homogeneous FTS state controller for the double integrator under rather restrictive conditions on parameters of the controller. In [28] an output feedback control is proposed based on homogeneity techniques and on a sliding-mode observer. The approach proposed here relies on the theories of homogeneity and input-to-state stability in continuous systems [29], [32].

Second, the robustness properties of this output control algorithm are studied. It is shown in [28] that this control is robust with respect to disturbances bounded by a function of the output. Our objective in this work is to relax the applicability conditions for the control obtained in [30], and to improve robustness abilities of the FTS output control with respect to [28] with purely continuous controller and observer. The improvement idea is, again, based on the homogeneity framework application. Finally, the effects of the control discretization on the system stability is studied. It is shown that, provided that the sampling rate is small enough, practical stability is achieved, and a qualitative estimation of the asymptotically stable set is given.

The outline of this work is as follows. Notation and introduction of the FTS and the homogeneity concepts are given in Section 2. The precise problem formulation is presented in Section 3. The output FTS controller is designed in Section 4. The robustness and the influence of the discretization are studied in Section 5. The results of computer simulations of the proposed control algorithm are presented in Section 6.

\section{Preliminaries}

\section{A. Notation}

Through the paper the following notation will be used:

- $\mathbb{R}_{+}=\{x \in \mathbb{R}: x \geq 0\}$, where $\mathbb{R}$ is the set of real numbers.

- For any real number $\alpha \geq 0$ and for all $x \in \mathbb{R}$ we define $\lceil x\rfloor^{\alpha}=$ $\operatorname{sign}(x)|x|^{\alpha}$.

- A continuous function $\alpha: \mathbb{R}_{+} \rightarrow \mathbb{R}_{+}$belongs to the class $\mathcal{K}$ if $\alpha(0)=0$ and the function is strictly increasing; a class $\mathcal{K}$ function belongs to the class $\mathcal{K}_{\infty}$ if it is increasing to infinity.

- A continuous function $\beta: \mathbb{R}_{+} \times \mathbb{R}_{+} \rightarrow \mathbb{R}_{+}$belongs to the class $\mathcal{K} \mathcal{L}$ if $s \mapsto \beta(s, t)$ is a class $\mathcal{K}$ function for any fixed $t$ and $\beta(s, t)$ is decreasing to 0 when $t \rightarrow+\infty$ for any fixed $s$.

\section{B. Finite-time stabilization}

Let us consider the closed loop system

$$
\dot{x}=F(x),
$$

where $F$ is a continuous vector field.

Definition 1. [1] The origin of the system (1) is finite-time stable (FTS) iff there exists a neighborhood of the origin $\mathcal{V}$ such that:

1) For any $x_{0} \in \mathcal{V}$ there exists $t_{0} \geq 0$ such that for any solution $x(t)$ of (1) such that $x(0)=x_{0}$ we have $x(t)=0$ for all $t \geq t_{0}$. We denote $T\left(x_{0}\right)$ the infimum of all such $t_{0}$ and we call the function $T: \mathcal{V} \rightarrow \mathbb{R}_{+}$the settling-time function of the system (1).

2) For any neighborhood of the origin $\mathcal{U}_{1} \subset \mathcal{V}$, there exists a neighborhood of the origin $\mathcal{U}_{2}$ such that for any $x_{0} \in \mathcal{U}_{2}$ and any solution $x(t)$ of (1) such that $x(0)=x_{0}$ we have $x(t) \in \mathcal{U}_{1}$ for all $t \geq 0$. 
Moreover, if the neighborhood $\mathcal{V}$ can be chosen to be $\mathbb{R}^{n}$, then the origin of the system (1) is said to be globally finite-time stable (GFTS).

Assuming forward uniqueness of solutions and the continuity of the settling time function, Bhat and Bernstein (see [1, Definition 2.2]) showed that FTS of the origin is equivalent to the existence of a $C^{1}$ positive definite function $V$ defined on a neighborhood of the origin satisfying $\dot{V}(x) \leq-c V(x)^{a}$ with $a \in(0,1), c>0$. In order to circumvent the classical Lyapunov function art of design, one can use homogeneity conditions recalled below.

\section{Homogeneity}

Let $\mathbf{r}=\left(r_{1}, \ldots, r_{n}\right)$ be a $n$-uplet of positive real numbers, thereafter called a generalized weight. Then $\Lambda_{r} x=\left(\ldots, \lambda^{r_{i}} x_{i}, \ldots\right)$ for any positive number $\lambda$ represents a mapping $x \mapsto \Lambda_{\mathrm{r}} x$ usually called a dilation (see [8]).

Definition 2. A function $h: \mathbb{R}^{n} \rightarrow \mathbb{R}$ is r-homogeneous of degree $\kappa \in \mathbb{R}$ if for all $x \in \mathbb{R}^{n}$ and all $\lambda>0$ we have $h\left(\Lambda_{\mathbf{r}} x\right)=\lambda^{\kappa} h(x)$.

Definition 3. A vector field $F: \mathbb{R}^{n} \rightarrow \mathbb{R}^{n}$ is r-homogeneous of degree $\kappa$ if for all $x \in \mathbb{R}^{n}$ and all $\lambda>0$ we have $F\left(\Lambda_{r} x\right)=$ $\lambda^{\kappa} \Lambda_{r} F(x)$, or equivalently, if the coordinate functions $F_{i}$ are $\mathbf{r}$ homogeneous of degree $\kappa+r_{i}$. When such a property holds, the corresponding nonlinear ODE (1) is said to be $\mathbf{r}$-homogeneous of degree $\kappa$.

Among many properties of homogeneous systems, let us mention the following results that will be of great importance to demonstrate the qualitative properties of the systems studied throughout the paper.

Theorem 1. [5] Let $F$ be a continuous $\mathbf{r}$-homogeneous vector field on $\mathbb{R}^{n}$ of negative degree. If the origin is Locally Asymptotically Stable (LAS) then it is GFTS.

Theorem 2. [5] Let $F$ be a continuous $\mathbf{r}$-homogeneous vector field on $\mathbb{R}^{n}$ of degree $\kappa \in \mathbb{R}$. If the origin is GAS, then for all $\mu>$ $\max \{0,-\kappa\}$ there exists a continuous positive definite function $V$ : $\mathbb{R}^{n} \rightarrow \mathbb{R}$, r-homogeneous of degree $\mu$ such that $V$ is $C^{1}$ on $\mathbb{R}^{n} \backslash\{0\}$ and for all $x \neq 0$ we have $d_{x} V F(x)<0$.

\section{PROBLEM FORMULATION}

Our contribution aims at designing a FTS output feedback based on homogeneity for the following double-integrator system

$$
\left\{\begin{array}{l}
\dot{x}_{1}=x_{2} \\
\dot{x}_{2}=u\left(x_{1}, x_{2}\right), \\
y=x_{1}
\end{array}\right.
$$

where $x_{1}$ and $x_{2}$ are the states of the system, $u$ is the input and $y$ is the output. We will proceed in four steps:

1) Design a homogeneous state feedback control ensuring GFTS for the double integrator

$$
\left\{\begin{array}{l}
\dot{x}_{1}=x_{2} \\
\dot{x}_{2}=u
\end{array}\right.
$$

2) Design a homogeneous observer

$$
\left\{\begin{array}{l}
\dot{\hat{x}}_{1}=\hat{x}_{2}-\chi_{1}\left(y-\hat{x}_{1}\right), \\
\dot{\hat{x}}_{2}=u-\chi_{2}\left(y-\hat{x}_{1}\right),
\end{array}\right.
$$

where $\chi_{1}$ and $\chi_{2}$ are functions to be designed such that the origin is GFTS for the error $e=x-\hat{x}$ equation:

$$
\left\{\begin{array}{l}
\dot{e}_{1}=e_{2}+\chi_{1}\left(e_{1}\right) \\
\dot{e}_{2}=\chi_{2}\left(e_{1}\right)
\end{array}\right.
$$

3) Show a separation principle such that the obtained observerbased closed loop system is GFTS

$$
\left\{\begin{array}{l}
\dot{x}_{1}=x_{2} \\
\dot{x}_{2}=u\left(y, \hat{x}_{2}\right) \\
y=x_{1}
\end{array},\right.
$$

where $\hat{x}_{2}$ is obtained from (4).

4) Study the robustness of the closed loop system and the influence of the discretization of the control and of the observer. Since this study is based on the results of [31], [32], which deal with continuous-time systems, continuous controller and observer are considered only.

\section{FINITE-TIME OUTPUT FEEDBACK BASED ON HOMOGENEITY}

\section{A. Finite-time control}

Let us consider the double integrator (3) with the following control

$$
u=k_{1}\left\lfloor x_{1}\right\rceil^{\frac{\alpha}{2-\alpha}}+k_{2}\left\lfloor x_{2}\right\rceil^{\alpha},
$$

with $\alpha \in[0,1]$. Let us mention that, letting $\alpha=0$, we recover the discontinuous system

$$
\left\{\begin{array}{l}
\dot{x}_{1}=x_{2} \\
\dot{x}_{2}=k_{1} \operatorname{sgn}\left(x_{1}\right)+k_{2} \operatorname{sgn}\left(x_{2}\right)
\end{array} .\right.
$$

Since we shall restrict ourselves to continuous systems, we will consider $\alpha>0$ and we let the reader refer to [33] and the references therein for a study of the case $\alpha=0$. On the other hand, taking $\alpha=1$, we recover a linear system. Hence, in all the sequel, we assume $\alpha \in(0,1)$.

The system (3) with the feedback (7) takes the form

$$
\left\{\begin{array}{l}
\dot{x}_{1}=x_{2} \\
\dot{x}_{2}=k_{1}\left\lfloor x_{1}\right\rceil^{\frac{\alpha}{2-\alpha}}+k_{2}\left\lfloor x_{2}\right\rceil^{\alpha} .
\end{array}\right.
$$

A direct verification shows that, taking $\mathbf{r}=(2-\alpha, 1)$, the system (9) is $\mathbf{r}$-homogeneous of degree $\alpha-1<0$.

Theorem 3. If $k_{1}<0$ and $k_{2}<0$ then the system (9) is GFTS.

Proof. Consider the following function

$$
V: x \mapsto \frac{-k_{1}(2-\alpha)}{2}\left|x_{1}\right|^{\frac{2}{2-\alpha}}+\frac{x_{2}^{2}}{2} .
$$

The function $V$ is continuously differentiable, proper, $\mathbf{r}$-homogeneous of degree 2 and $\dot{V}=k_{2}\left|x_{2}\right|^{1+\alpha}$. Since $k_{1}<0$ and $k_{2}<0$, the function $V$ is definite positive, and $\dot{V}$ is negative semi-definite. A direct application of the LaSalle invariance principle shows that the origin is GAS for the system (9). Being homogenous of negative degree, the system (9) is therefore GFTS by Theorem 1.

Remark 1. In [30] these conditions have been obtained for $\alpha$ sufficiently close to one.

This result was also proven in [28] under the additional assumption $k_{1}<k_{2}$, which is only necessary when considering $\alpha=0$.

\section{B. Finite-time observer design}

A finite-time observer for a canonical observable form was constructed for the first time in [17]. Similar ideas were used in [28] for designing a discontinuous finite-time observer. In both cases, the proof of finite-time stability was based on homogeneity. In the case of the double integrator, the observer of [17] is

$$
\left\{\begin{array}{l}
\dot{\hat{x}}_{1}=\hat{x}_{2}-l_{1}\left\lfloor y-\hat{x}_{1}\right\rceil^{\beta} \\
\dot{\hat{x}}_{2}=u-l_{2}\left\lfloor y-\hat{x}_{1}\right\rceil^{2 \beta-1}
\end{array},\right.
$$

with $\beta \in\left(\frac{1}{2}, 1\right)$. 
The error dynamics can be written as follows

$$
\left\{\begin{array}{l}
\dot{e}_{1}=e_{2}+l_{1}\left\lfloor e_{1}\right\rceil^{\beta} \\
\dot{e}_{2}=l_{2}\left\lfloor e_{1}\right\rceil^{2 \beta-1}
\end{array} .\right.
$$

where $e=x-\hat{x}$ and the right hand side is $\rho$-homogeneous of degree $\rho_{1}(\beta-1)$ where $\rho=\left(\rho_{1}, \rho_{1} \beta\right)$.

When taking $\beta=1$, we recover a linear equation. When taking $\beta=1 / 2$, we recover a particular case of the discontinuous observer from [28] and we will again omit this case to restrict ourselves to continuous systems. In [17], the FTS of the system (12) was proved for $\beta \in(1-\varepsilon, 1)$ for a sufficiently small $\varepsilon>0$. Here we shall prove that the system is FTS for all $\beta \in\left(\frac{1}{2}, 1\right)$ and all $\rho_{1}>0$.

Theorem 4. The observer (11) with $\chi_{1}\left(e_{1}\right)=l_{1}\left\lfloor e_{1}\right\rceil^{\beta}, \chi_{2}\left(e_{1}\right)=$ $l_{2}\left\lfloor e_{1}\right\rceil^{2 \beta-1}$ is GFTS in the coordinates $\left(e_{1}, e_{2}\right)$ for any $\beta \in\left(\frac{1}{2}, 1\right)$, and for any $l_{1}<0$ and $l_{2}<0$.

Proof. Consider the following function

$$
V(e)=-\frac{l_{2}}{2 \beta}\left|e_{1}\right|^{2 \beta}+\frac{e_{2}^{2}}{2} .
$$

The function $V$ is positive definite, proper, continuously differentiable and homogeneous with degree $2 \rho_{1} \beta$. Moreover, we compute $\dot{V}(e)=$ $-l_{1} l_{2}\left|e_{1}\right|^{3 \beta-1} \leq 0$. By the LaSalle invariance principle, we easily prove that the system (12) is GAS. Being homogeneous, this system is therefore GFTS by Theorem 1.

Thus the observer (11) ensures observation of the state of the system (2) in a finite time for any initial condition.

\section{Finite-time stable observer based control}

Our aim is now to use the two preceeding subsections to build a finite-time observer based control. In view of Theorem 3, we assume here that $k_{1}<0$ and $k_{2}<0$. Let us rewrite the system (6) for the designed FTS control (7) and the FTS observer (12) (in the estimation error coordinates)

$$
\left\{\begin{array}{l}
\dot{x}_{1}=x_{2} \\
\dot{x}_{2}=k_{1}\left\lfloor x_{1}\right\rceil^{\frac{\alpha}{2-\alpha}}+k_{2}\left\lfloor x_{2}-e_{2}\right\rceil^{\alpha} \\
\dot{e}_{1}=e_{2}+l_{1}\left\lfloor e_{1}\right\rceil^{\beta} \\
\dot{e}_{2}=l_{2}\left\lfloor e_{1}\right\rceil^{2 \beta-1}
\end{array} .\right.
$$

Remark 2. Note that $x_{2}-e_{2}=\hat{x}_{2}$, thus the control depends on the measured output $x_{1}$ only. Moreover, we could replace $x_{1}$ in this equation by $\hat{x}_{1}=x_{1}-e_{1}$ without changing the following results.

To prove the FTS property of this system we need two auxiliary lemmas.

Lemma 1. For $\theta \in(0,1)$, the function $a \mapsto\lfloor a\rceil^{\theta}$ is $\theta$-Hölder on $\mathbb{R}$ with corresponding constant $2^{1-\theta}$. In particular, for all $e_{2} \in \mathbb{R}$, and all $x_{2} \in \mathbb{R}$ we have $\left|\left\lfloor x_{2}-e_{2}\right\rceil^{\alpha}-\left\lfloor x_{2}\right\rceil^{\alpha}\right| \leq 2^{1-\alpha}\left|e_{2}\right|^{\alpha}$.

Proof. Define for $a, b \in \mathbb{R}$ and $\theta \in(0,1)$

$$
g_{\theta}(a, b)=\lfloor a+b\rceil^{\theta}-\lfloor a\rceil^{\theta} .
$$

Let us show that $\left|g_{\theta}(a, b)\right| \leq 2^{1-\theta}|b|^{\theta}$, which will prove the lemma. It is clear that this inequality is true for $b=0$. In the sequel, we assume $b \neq 0$. An easy verification shows that for all $\lambda>0$

$$
\begin{aligned}
g_{\theta}(\lambda a, \lambda b) & =\lambda^{\theta} g_{\theta}(a, b), \\
g_{\theta}(a, b) & =\lfloor b\rceil^{\theta} g_{\theta}\left(\frac{a}{b}, 1\right) .
\end{aligned}
$$

Let us denote $h_{\theta}: z \in \mathbb{R} \mapsto g_{\theta}(z, 1)$. The function $h_{\theta}$ is differentiable for all $z \notin\{-1,0\}$ and $h_{\theta}^{\prime}(z)=\theta\left(|1+z|^{\theta-1}-|z|^{\theta-1}\right)$. We easily show that $h$ is strictly increasing on $(-\infty,-1 / 2)$ and strictly decreasing on $(-1 / 2,+\infty)$. Thus, we find $0 \leq h(z) \leq h(-1 / 2)=$ $2^{1-\theta}$. Finally, we have $g_{\theta}(a, b)=\lfloor b\rceil^{\theta} g_{\theta}\left(\frac{a}{b}, 1\right)=\lfloor b\rceil^{\theta} h_{\theta}\left(\frac{a}{b}\right)$, and therefore $\left|g_{\theta}(a, b)\right| \leq 2^{1-\theta}|b|^{\theta}$.

Lemma 2. The system

$$
\left\{\begin{array}{l}
\dot{x}_{1}=x_{2} \\
\dot{x}_{2}=k_{1}\left\lfloor x_{1}\right\rceil^{\frac{\alpha}{2-\alpha}}+k_{2}\left\lfloor x_{2}-e_{2}\right\rceil^{\alpha}
\end{array}\right.
$$

is Input-to-State Stable (ISS) with respect to the input $e_{2}$.

Input-to-State Stability and other related properties were introduced in [34]. This ISS property was used in [35] for designing finite-time control laws. The ISS property of homogeneous systems has been already studied in [36], [29]. In [36] a general nonlinear homogeneous system is studied with degree greater than or equal to 1 ; in [29] the degree restriction has been relaxed, but it was assumed that the system dynamics depends linearly on the disturbance. Definitions and properties of ISS systems can be found in these references. In recent works [31], [32] these constraints have been relaxed and an extension to integral ISS was proposed. The lemma is a corollary of Theorem 6 from [32].

We are now in position to formulate the main result of this section.

Theorem 5. The system (13) is GFTS for any $\alpha \in(0,1)$ and $\beta \in$ $(1 / 2,1)$ for any $k_{1}<0, k_{2}<0, l_{1}<0$ and $l_{2}<0$.

Proof. By the stability of the observer and the ISS of the state equation, there exists $\gamma \in \mathcal{K}$ and $\alpha, \beta \in \mathcal{K} \mathcal{L}$ such that for any $t_{0} \geq 0$ and all $t \geq t_{0}$

$$
\begin{aligned}
\|e(t)\| & \leq \alpha\left(\left\|e\left(t_{0}\right)\right\|, t-t_{0}\right), \\
\|x(t)\| & \leq \beta\left(\left\|x\left(t_{0}\right)\right\|, t-t_{0}\right)+\gamma\left(\sup _{\tau \in\left[t_{0}, t\right]}\|e(\tau)\|\right) .
\end{aligned}
$$

We obviously have the estimates $\sup _{\tau>0}\|e(\tau)\| \leq \alpha(\|e(0)\|, 0)$ and then $\|x(t)\| \leq \beta(\|x(0)\|, 0)+\gamma(\alpha(\|e(0)\|, 0))$. Finally, denoting $\|(x, e)\|=\|x\|+\|e\|$ we find

$$
\|(x(t), e(t))\| \leq \beta(\|x(0)\|, 0)+\alpha(\|e(0)\|, 0)+\gamma(\alpha(\|e(0)\|, 0))
$$

which gives the stability.

The finite-time convergence of the system is a direct consequence of the finite-time convergence of the error $e$ and the finite-time convergence of the system (9). We conclude that the system (13) is GFTS.

Remark 3. It is worth to stress that the system (13) is FTS in coordinates $\left(e_{1}, e_{2}\right)$ (see Theorem 4 ) and it is FTS in coordinates $\left(x_{1}, x_{2}, e_{1}, e_{2}\right)$ (Theorem 5). Moreover, taking $\hat{x}(0)=0$, we find $e(0)=x(0)$ and hence

$$
\|x(t)\| \leq \beta(\|x(0)\|, t)+\gamma(\alpha(\|x(0)\|, 0)) .
$$

This actually proves the stability of the isolated coordinates $\left(x_{1}, x_{2}\right)$ provided that we choose $\hat{x}(0)=0$.

Finally, let us mention that results similar to Theorems 4 and 5 were proved in [21] using a different proof methodology.

\section{ROBUSTNESS PROPERTIES OF THE CLOSED LOOP SYSTEM AND EFFECTS OF THE DISCRETIZATION}

The output feedback given in Section III has been studied, under slightly different forms, in the literature. We shall now go into the main part of this paper: the robustness of the system under the proposed output feedback and, particularly, the effects of the sampling on the stability.

If we choose $\beta=\frac{1}{2-\alpha}$ and $\rho_{1}=2-\alpha$ in (13), it is easy to see that the system (13) becomes $\mathbf{R}$-homogeneous of degree $\alpha-1$ where $\mathbf{R}=\left(r_{1}, r_{2}, \rho_{1}, \rho_{2}\right)=(2-\alpha, 1,2-\alpha, 1)$. This choice provides 
another proof of Theorem 5 without the help of the ISS property: thanks to homogeneity, the attractiveness of the origin implies its stability.

In this section, we will study the robustness properties that we can get in this setting. Indeed, we will be interested in the system

$$
\left\{\begin{array}{l}
\dot{x}_{1}=x_{2} \\
\dot{x}_{2}=k_{1}\left\lfloor x_{1}\right\rceil^{\frac{\alpha}{2-\alpha}}+k_{2}\left\lfloor x_{2}-e_{2}\right\rceil^{\alpha} \\
\dot{e}_{1}=e_{2}+l_{1}\left\lfloor e_{1}\right\rceil^{\frac{1}{2-\alpha}} \\
\dot{e}_{2}=l_{2}\left\lfloor e_{1}\right\rceil^{\frac{\alpha}{2-\alpha}}
\end{array} .\right.
$$

Assume that the system (14) is subject to disturbances:

1) a noise $d_{1}$ on the output $x_{1}$;

2) a perturbation $d_{2}$ which may appear in the transmission channel between the controller and the observer;

3) physical perturbations $d_{3}$ like frictions or unmodelled dynamics;

4) computationnal errors $\hat{d}_{1}$ and $\hat{d}_{2}$ on $\hat{x}_{1}$ and $\hat{x}_{2}$.

The disturbed system is now

$$
\left\{\begin{array}{l}
\dot{x}_{1}=x_{2} \\
\dot{x}_{2}=k_{1}\left\lfloor x_{1}+d_{1}\right\rceil^{\frac{\alpha}{2-\alpha}}+k_{2}\left\lfloor x_{2}-e_{2}+d_{2}+\hat{d}_{2}\right\rceil^{\alpha}+d_{3} \\
\dot{e}_{1}=e_{2}-\hat{d}_{2}+l_{1}\left\lfloor e_{1}-\hat{d}_{1}+d_{1}\right\rceil^{\frac{1}{2-\alpha}} \\
\dot{e}_{2}=l_{2}\left\lfloor e_{1}-\hat{d}_{1}+d_{1}\right\rceil^{\frac{\alpha}{2-\alpha}}+d_{3}
\end{array}\right.
$$

Let us denote the disturbance $\mathbf{d}=\left(d_{1}, d_{2}, d_{3}, \hat{d}_{1}, \hat{d}_{2}\right)$.

We have the following robustness result:

Theorem 6. Under the conditions of Theorem 5, the system (15) is ISS with respect to the input $\mathbf{d}$.

Proof. This claim follows a direct application of the results from Theorem 6 of [32].

This result states that some stability properties pertain for the system (14) under the aforementioned disturbances. Indeed, if these perturbations are bounded, practical stability ${ }^{1}$ is achieved. In addition, the shape of asymptotic gain function has also been evaluated in [32] based on the homogeneity arguments, and if the input $\mathbf{d}$ admits smallgain conditions, then GFTS property can be preserved for (15) that is an improvement of [28] (where a similar result has been proven for $\mathbf{d}=d_{3}$ only).

Similarly, we can study the influence of the discretization of the control and the observer in our observer-based feedback. We assume that there exists a sequence of times $\left(t_{k}\right)_{k \in \mathbb{N}}$ increasing to $+\infty$ at which the observer and the control are updated, such that $0<t_{k+1}-$ $t_{k} \leq h$. For $t \in\left(t_{k}, t_{k+1}\right)$, the observer and the control remain constant. The system can be rewritten, for $t \in\left[t_{k}, t_{k+1}\right)$

$$
\left\{\begin{aligned}
\dot{x}_{1}(t)= & x_{2}(t) \\
\dot{x}_{2}(t)= & k_{1}\left\lfloor x_{1}\left(t_{k}\right)\right\rceil^{\frac{\alpha}{2-\alpha}}+k_{2}\left\lfloor\hat{x}_{2}\left(t_{k}\right)\right\rceil^{\alpha} \\
\hat{x}_{1}\left(t_{k+1}\right)= & \hat{x}_{1}\left(t_{k}\right)+\left(t_{k+1}-t_{k}\right) \times \\
& \left(\hat{x}_{2}\left(t_{k}\right)-l_{1}\left\lfloor x_{1}\left(t_{k}\right)-\hat{x}_{1}\left(t_{k}\right)\right\rceil^{\frac{1}{2-\alpha}}\right) . \\
\hat{x}_{2}\left(t_{k+1}\right)= & \hat{x}_{2}\left(t_{k}\right)+\left(t_{k+1}-t_{k}\right) \times \\
& \left(u\left(t_{k}\right)-l_{2}\left\lfloor x_{1}\left(t_{k}\right)-\hat{x}_{1}\left(t_{k}\right)\right\rceil^{\frac{\alpha}{2-\alpha}}\right)
\end{aligned}\right.
$$

To compare this discrete system with the continuous system (14), we need to define some other variables. We define, for $t \in\left[t_{k}, t_{k+1}\right)$

$$
\left\{\begin{array}{l}
\dot{\tilde{x}}_{1}(t)=\hat{x}_{2}\left(t_{k}\right)-l_{1}\left\lfloor x_{1}\left(t_{k}\right)-\hat{x}_{1}\left(t_{k}\right)\right\rceil^{\frac{1}{2-\alpha}} \\
\dot{\tilde{x}}_{2}(t)=u\left(t_{k}\right)-l_{2}\left\lfloor x_{1}\left(t_{k}\right)-\hat{x}_{1}\left(t_{k}\right)\right\rceil^{\frac{\alpha}{2-\alpha}}
\end{array} .\right.
$$

${ }^{1} \mathrm{~A}$ system $\dot{x}=f(x)$ is practically stable if there exists an asymptotically stable compact set.
Setting $\tilde{x}_{1}\left(t_{0}\right)=\hat{x}_{1}\left(t_{0}\right)$ and $\tilde{x}_{2}\left(t_{0}\right)=\hat{x}_{2}\left(t_{0}\right)$ leads to $\tilde{x}_{1}\left(t_{k}\right)=$ $\hat{x}_{1}\left(t_{k}\right)$ and $\tilde{x}_{2}\left(t_{k}\right)=\hat{x}_{2}\left(t_{k}\right)$ for any $k \in \mathbb{N}$. These variables are affine interpolations of the discrete system. We are naturally led to define new "observation errors" by $\varepsilon_{1}=x_{1}-\tilde{x}_{1}$ and $\varepsilon_{2}=x_{2}-\tilde{x}_{2}$. Finally, setting $\pi(t)=\max \left\{t_{k}, t_{k} \leq t\right\}$ and

$$
\left\{\begin{array}{l}
d_{1}(t)=x_{1}\left(t_{k}\right)-x_{1}(t)=x_{1}(\pi(t))-x_{1}(t) \\
\tilde{d}_{2}(t)=\tilde{x}_{2}\left(t_{k}\right)-\tilde{x}_{2}(t)=\tilde{x}_{2}(\pi(t))-\tilde{x}_{2}(t) \\
\tilde{d}_{1}(t)=\tilde{x}_{1}\left(t_{k}\right)-\tilde{x}_{1}(t)=\tilde{x}_{1}(\pi(t))-\tilde{x}_{1}(t)
\end{array},\right.
$$

we get, for $t \in \mathbb{R}_{+}$

$$
\left\{\begin{array}{l}
\dot{x}_{1}=x_{2} \\
\dot{x}_{2}=k_{1}\left\lfloor x_{1}+d_{1}\right\rceil^{\frac{\alpha}{2-\alpha}}+k_{2}\left\lfloor x_{2}-\varepsilon_{2}+\tilde{d}_{2}\right\rceil^{\alpha} \\
\dot{\varepsilon}_{1}=\varepsilon_{2}-\tilde{d}_{2}+l_{1}\left\lfloor\varepsilon_{1}-\tilde{d}_{1}+d_{1}\right\rceil^{\frac{1}{2-\alpha}} \\
\dot{\varepsilon}_{2}=l_{2}\left\lfloor\varepsilon_{1}-\tilde{d}_{1}+d_{1}\right\rceil^{\frac{\alpha}{2-\alpha}}
\end{array} .\right.
$$

Therefore, setting $z=\left(x_{1}, x_{2}, \varepsilon_{1}, \varepsilon_{2}\right)$ and $\Delta=\left(d_{1}, \tilde{d}_{1}, \tilde{d}_{2}\right)$, Theorem 6 yields that the system (19) is ISS w.r.t. the input $\Delta$. But we can actually characterize this property more precisely. Let us denote $\tilde{N}(\Delta)=\left|d_{1}\right|^{\frac{1}{2-\alpha}}+\left|\tilde{d}_{1}\right|^{\frac{1}{2-\alpha}}+\left|d_{2}\right|$. The function $\tilde{N}$ is $\tilde{\mathbf{R}}$-homogeneous of degree 1 with $\tilde{\mathbf{R}}=(2-\alpha, 2-\alpha, 1)$.

Proposition 1. Consider a R-homogeneous Lyapunov function $V$ of degree $\mu$ for the $\mathbf{R}$-homogeneous system (14), as given by Theorem 2. There exists a constant $C_{1}>0$ such that the solutions $z(t)$ of system (19) with input $\Delta(t)$ verify:

$$
V(z(t)) \leq \max \left\{\beta(V(z(0)), t) ; C_{1}^{\mu} \sup _{\tau \in[0, t]} \tilde{N}(\Delta(\tau))^{\mu}\right\}, \quad \forall t \geq 0
$$

with $\beta$ is a class $\mathcal{K} \mathcal{L}$ function.

Proof. Let us denote

$$
F(z, \Delta)=\left(\begin{array}{c}
x_{2} \\
k_{1}\left\lfloor x_{1}+d_{1}\right\rceil^{\frac{\alpha}{2-\alpha}}+k_{2}\left\lfloor x_{2}-\varepsilon_{2}+\tilde{d}_{2}\right\rceil^{\alpha} \\
\varepsilon_{2}-\tilde{d}_{2}+l_{1}\left\lfloor\varepsilon_{1}-\tilde{d}_{1}+d_{1}\right\rceil^{\frac{1}{2-\alpha}} \\
l_{2}\left\lfloor\varepsilon_{1}-\tilde{d}_{1}+d_{1}\right\rceil^{\frac{\alpha}{2-\alpha}}
\end{array}\right) .
$$

Consider $z \neq 0$ and denote $\lambda=V(z)^{1 / \mu}$ and $\zeta=\Lambda_{\mathbf{R}}^{-1} z$. We have $V(\zeta)=1$. Finally, let us denote $-a=\sup _{V(w)=1} d_{w} V F(w, 0)<0$ and $b=\sup _{V(w)=1}\left\|d_{w} V\right\|>0$. We have

$$
\begin{aligned}
d_{z} V F(z, \Delta)= & \lambda^{\alpha-1+\mu} d_{\zeta} V F\left(\zeta, \Lambda_{\tilde{\mathbf{R}}}^{-1} \Delta\right) \\
= & V(z)^{\frac{\alpha-1+\mu}{\mu}}\left[d_{\zeta} V F(\zeta, 0)+\right. \\
& \left.d_{\zeta} V\left(F\left(\zeta, \Lambda_{\tilde{\mathbf{R}}}^{-1} \Delta\right)-F(\zeta, 0)\right)\right] \\
\leq & \left.V(z)^{\frac{\alpha-1+\mu}{\mu}}\left[-a+b \| F\left(\zeta, \Lambda_{\tilde{\mathbf{R}}}^{-1} \Delta\right)-F(\zeta, 0)\right) \|\right]
\end{aligned}
$$

By continuity of $F$, there exists $\varepsilon>0$ such that if $\tilde{N}(\Delta)<\varepsilon$ then $\left.\sup _{V(\zeta)=1} \| F(\zeta, \Delta)-F(\zeta, 0)\right) \|<\frac{a}{2 b}$. Hence, if $\tilde{N}\left(\Lambda_{\tilde{\mathbf{R}}}^{-1} \Delta\right)<\varepsilon$ we find that

$$
d_{z} V F(z, \Delta) \leq-\frac{a}{2 b} V(z)^{\frac{\alpha-1+\mu}{\mu}} .
$$

That is (20) holds as long as $\lambda \geq \tilde{N}(\Delta) / \varepsilon$ or, equivalently, we have $V(z(t)) \leq \beta(V(z(0)), t)$ as long as $V(z) \geq C_{1}^{\mu} \tilde{N}(\Delta)^{\mu}$, where $\beta$ is a class $\mathcal{K} \mathcal{L}$ function given by the integration of (20) and $C_{1}=1 / \varepsilon$. The announced inequality follows. 
Let us now study the variations of the input $\Delta$ through time.

$$
\begin{aligned}
\left|d_{1}(t)\right| & =\left|\int_{\pi(t)}^{t} \dot{x_{1}}(\tau) d \tau\right| \\
& \leq \int_{\pi(t)}^{t}\left|x_{2}(\tau)-x_{2}(\pi(t))\right| d \tau+h\left|x_{2}(\pi(t))\right| \\
& \leq \int_{\pi(t)}^{t} \int_{\pi(t)}^{\tau}|u(\pi(t))| d s d \tau+h\left|x_{2}(\pi(t))\right| \\
& \leq h^{2}|u(\pi(t))|+h\left|x_{2}(\pi(t))\right|
\end{aligned}
$$

where $u(\pi(t))=k_{1}\left\lfloor x_{1}(\pi(t))\right\rceil^{\frac{\alpha}{2-\alpha}}+k_{2}\left\lfloor\tilde{x}_{2}(\kappa(t))\right\rceil^{\alpha}$. Similarly, we get

$$
\begin{aligned}
& \left|\tilde{d}_{2}(t)\right| \leq h\left|u(\pi(t))-l_{1}\left\lfloor\varepsilon_{1}(\pi(t))\right\rceil^{\frac{1}{2-\alpha}}\right| \\
& \left|\tilde{d}_{1}(t)\right| \leq h\left|\tilde{x}_{2}(\pi(t))-l_{2}\left\lfloor\varepsilon_{1}(\pi(t))\right\rceil^{\frac{\alpha}{2-\alpha}}\right| .
\end{aligned}
$$

In the sequel, for the sake of simplicity, we assume that $h \leq 1$. Using classical arguments of homogeneous functions comparison (see for instance [30]), we deduce that there exists a constant $C_{2}>0$ such that, denoting

$$
N(z)=V(z)^{1 / \mu},
$$

we have $\tilde{N}(\Delta(t)) \leq \gamma_{2}(N(\pi(t)))$, where

$$
\gamma_{2}(s)=C_{2} h^{\frac{1}{2-\alpha}}\left\{\begin{array}{lll}
s^{\frac{\alpha}{2-\alpha}} & \text { if } & s \leq 1 \\
s & \text { if } & s \geq 1
\end{array} .\right.
$$

The purpose of the consideration below it to prove that the system (19) is practically stable and converging to a ball, which radius is a class $\mathcal{K}$ of $h$, provided that the following inequality holds

$$
h<\left(C_{1} C_{2}\right)^{\alpha-2} .
$$

Denote $\theta(s)=s-C_{1} \gamma_{2}(s)$ and

$$
s_{h}=\left(C_{1} C_{2}\right)^{\frac{2-\alpha}{2-2 \alpha}} h^{\frac{1}{2-2 \alpha}} .
$$

Lemma 3. For all $h>0$ such that the condition (22) holds, the function $\theta$ is strictly increasing for $s>s_{h}, \theta\left(s_{h}\right)=0$ and $\theta(s) \rightarrow$ $+\infty$ when $s \rightarrow+\infty$.

Proof. Let us distinguish 2 cases

- if $s \geq 1, \gamma_{2}(s)=C_{2} h^{\frac{1}{2-\alpha}} s$ and hence $\theta(s)=\left(1-C_{1} C_{2} h\right) s$ with $1-C_{1} C_{2} h>0$, thus $\theta$ is strictly increasing, positive and tends to infinity.

- if $s \leq 1, \gamma_{2}(s)=C_{2} h^{\frac{1}{2-\alpha}} s^{\frac{\alpha}{2-\alpha}}$ and hence we have $\theta(s)=$ $s^{\frac{\alpha}{2-\alpha}}\left(s^{\frac{2-2 \alpha}{2-\alpha}}-C_{1} C_{2} h^{\frac{1}{2-\alpha}}\right)$. It is clear that the function is positive and strictly increasing for $s>s_{h}$.

Theorem 7. Under the conditions of Theorem 5, if (22) holds, then the set $K=\left\{N(z) \leq s_{h}\right\}$, which is a compact neighborhood of the origin, is globally asymptotically stable for the system (16) with $s_{h}$ given by (23) and $N$ given by (21).

Proof. Let us first show the stability. By the Proposition 1 and the preceding discussion we have

$N(z(t)) \leq \beta_{0}\left(N\left(z\left(t_{0}\right)\right), t-t_{0}\right)+C_{1} \sup _{\tau \in\left[t_{0}, t\right]} \gamma_{2}(N(\pi(\tau))), \forall t \geq t_{0}$, with $\beta_{0}$ a class $\mathcal{K} \mathcal{L}$ function. Since $\pi(t) \leq t$ and $\gamma_{2} \in \mathcal{K}$, we have $N(z(t)) \leq \beta_{0}\left(N\left(z\left(t_{0}\right)\right), t-t_{0}\right)+C_{1} \gamma_{2}\left(\sup _{\tau \in\left[t_{0}, t\right]} N(\tau)\right), \quad \forall t \geq t_{0}$.

Let $t_{\max }$ belongs to the interval of definition of $z(t)$, for $t \in$ $\left[0, t_{\max }\right]$ we have

$$
N(z(t)) \leq \beta_{0}(N(z(0)), 0)+C_{1} \gamma_{2}\left(\sup _{\tau \in\left[0, t_{\max }\right]} N(\tau)\right),
$$

and thus $\theta\left(\sup _{\tau \in\left[0, t_{\max }\right]} N(z(\tau))\right) \leq \beta_{0}(N(y(0)), 0)$. By Lemma 3 , the function $\tilde{\theta}: \sigma \mapsto \theta\left(\sigma+s_{h}\right)$ is a class $\mathcal{K}$ function. Hence we get that $\sup _{\tau \in\left[0, t_{\max }\right]} N(z(\tau)) \leq s_{h}+\tilde{\theta}^{-1}\left(\beta_{0}(N(z(0)), 0)\right)$. This inequality being true for all $t_{\max }$, it yields that

$$
N(z(\tau)) \leq s_{h}+\tilde{\theta}^{-1}\left(\beta_{0}(N(z(0)), 0)\right) \quad \forall t \geq 0
$$

that is, the set $K$ is stable.

Let us now prove that $\lim \sup _{t \rightarrow \infty} N(z(t)) \leq s_{h}$. The function $\beta_{0}$ being of class $\mathcal{K} \mathcal{L}$, for all $\varepsilon>0$ there exists $T_{0} \geq 0$ such that for all $t-t_{0} \geq T_{0}$, we have $\beta_{0}\left(N(z(0)), t-t_{0}\right) \leq \varepsilon$. Therefore, for all $t \geq t_{0}+T_{0}$

$$
\begin{aligned}
N(z(t)) & \leq \varepsilon+C_{1} \gamma_{2}\left(\sup _{\tau \geq t_{0}} N(z(\tau))\right) \\
\sup _{\tau \geq t_{0}+T_{0}} N(z(t)) & \leq \varepsilon+C_{1} \gamma_{2}\left(\sup _{\tau \geq t_{0}} N(z(\tau))\right) \\
\lim _{t_{0} \rightarrow+\infty} \sup _{\tau \geq t_{0}+T_{0}} N(z(t)) & \leq \varepsilon+C_{1} \gamma_{2}\left(\lim _{t_{0} \rightarrow+\infty} \sup _{\tau \geq t_{0}} N(z(\tau))\right) \\
\limsup _{t \rightarrow \infty} N(z(t)) & \leq \varepsilon+C_{1} \gamma_{2}\left(\limsup _{t \rightarrow \infty} N(z(\tau))\right) \\
\theta\left(\limsup _{t \rightarrow \infty} N(z(t))\right) & \leq \varepsilon .
\end{aligned}
$$

This last inequality is true for any $\varepsilon>0$, therefore we have $\theta\left(\limsup \sup _{t \rightarrow \infty} N(z(t))\right) \leq 0$ and thus $\lim \sup _{t \rightarrow \infty} N(z(t)) \leq s_{h}$ by Lemma 3 .

Theorem 7 is qualitative, it proves that, provided that the step $h$ is small enough, there exists a constant $C>0$ such that the state of the system converges to the set $K=\left\{N \leq C h^{\frac{1}{2-2 \alpha}}\right\}$. This fact has two consequences. The first one is purely theoretical: the discretized system is practically stable. This information is interesting because it ensures us that the state of the system will not blow up and furthermore will reach a neighborhood of the desired equilibrium. However, in practice, this information is not sufficient if we do not have an estimation on the neighborhood. But Theorem 7 actually yields another information. The asymptotically stable set has a particular shape, in fact a homogeneous ball, which radius is proportional to a power of the sampling step. The proportionality constant $C$ is unknown, but it can be evaluated via a numerical estimation technique. For instance, assume that we know an estimation of the asymptotically stable set for a given step $h_{0}$. Given that $K$ is a homogeneous ball with radius proportional to a power of $h$, we can deduce from our estimation the shape taken by the asymptotically stable set under a change of sampling step. For instance, if we sample twice faster, the radius of the homogeneous ball of convergence will be divided by $2^{\frac{1}{2-2 \alpha}}$. We can also conversely compute a step such that the attracting set is inside a given ball in the state space. By the way, we remark that the increasing the value of $\alpha$ implies shrinking the size of $K$. Hence, the parameter $\alpha$ should be selected according to the desired behavior of the system.

Let us finally mention that the theory of homogeneity allowed us to circumvent the explicit construction of a Lyapunov function. The results are demonstrated using qualitative methods and the properties that we have proved are hence qualitative. Up to now, no homogeneous Lyapunov function is known for the system (14), although Theorem 2 ensures us that such a function exists. But if, in the future, such a function happened to be found, the constant $C$ could be numerically estimated. Indeed, the constant $C_{2}$ can already be written as a function of the gains $k_{1}, k_{2}, l_{1}$ and $l_{2}$ and of the constant $\alpha$, while the construction of the constant $C_{1}$ is given in the proof of the Proposition 1. Doing this would turn Theorem 7 from a qualitative to a quantitative result. 

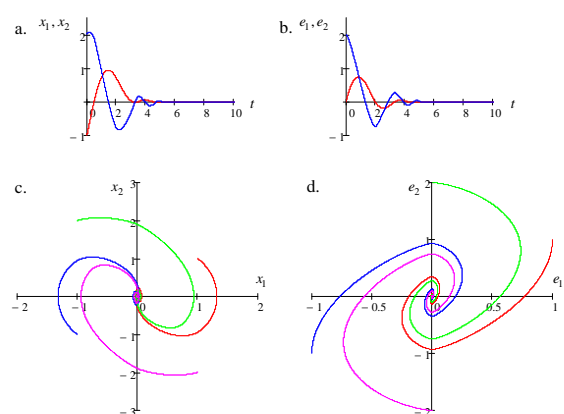

Figure 1. The results of simulation without disturbances, $h=0.002$
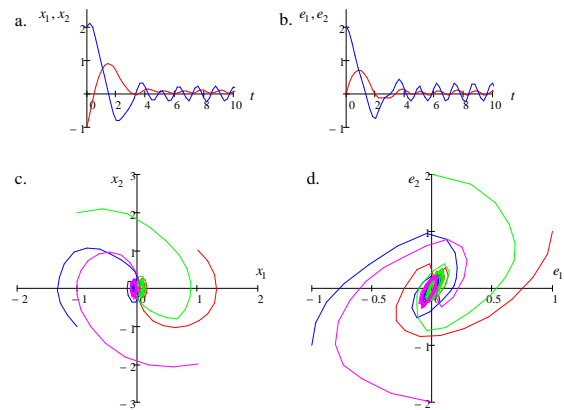

Figure 2. The results of simulation with disturbances, $h=0.2$

\section{Simulations}

Select $\alpha=0.6, \beta=\frac{1}{2-\alpha}$ and $k_{1}=-1, k_{2}=-2, l_{1}=-1$, $l_{2}=-2$, then clearly the conditions of Theorems 5 are satisfied.

The results of the system simulation are presented in figures 1,2 . In figures 1.a, 2.a and 1.b, 2.b the examples of transients in time are given for the system state $\left(x_{1}, x_{2}\right)$ and the estimation error $\left(e_{1}, e_{2}\right)$ respectively. In the case of Fig. 1 all disturbances are selected to be zero, the step of simulation $h=0.002$. In the case of Fig. $2 d_{1}(t)=$ $0.1 \sin (5 t)$ and $d_{3}(t)=0.1 \cos (6 t)$ with $h=0.2$ (the disturbances $d_{2}(t), \tilde{d}_{1}(t)$ and $\tilde{d}_{2}(t)$ are generated by the computational procedure used for simulation). As we can conclude from the results presented in Fig. 1, the system is converging to zero in a finite time for both pairs of variables, and the convergence is also monotone (that justifies the theoretical results obtained above). From Fig. 2 we see that the trajectories stay bounded in the presence of disturbances and that they converge to some ball around the origin even for a rather large simulation step $h$.

\section{CONCLUSION}

The problems of finite-time control and state estimation for the double integrator are studied. A finite-time output control is designed. An extension of applicability conditions of the homogeneous control algorithm from [11] is obtained. An improved robustness of the proposed output control with respect to the result of [28] is proven. It is shown that discretization does not destroy stability of the presented control algorithm. The efficiency of the obtained solution is demonstrated by computer simulations.

Development of the approach to the case of $n^{\text {th }}$-dimensional integrator and evaluation of the settling time function are the possible future directions of the research.

\section{REFERENCES}

[1] S. Bhat and D. Bernstein, "Finite time stability of continuous autonomous systems," SIAM J. Control Optim., vol. 38, no. 3, pp. 751-766, 2000.

[2] V. Haimo, "Finite time controllers," SIAM J. Control Optim., vol. 24, no. 4, pp. 760-770, 1986.

[3] E. Moulay and W. Perruquetti, "Finite time stability of non linear systems," in IEEE Conference on Decision and Control, (Hawaii, USA), pp. 3641-3646, 2003.

[4] Y. Orlov, "Finite time stability and robust control synthesis of uncertain switched systems," SIAM J. Control Optim., vol. 43, no. 4, pp. 12531271, 2005.

[5] L. Rosier, Etude de Quelques Problèmes de Stabilisation. PhD thesis, Ecole Normale Supérieure de Cachan, 1993.

[6] A. Bacciotti and L. Rosier, Lyapunov Functions and Stability in Control Theory. Springer, 2nd ed., 2005.

[7] S. Bhat and D. Bernstein, "Finite-time stability of homogeneous systems," in Proceedinds of the American Control Conference, (Albuquerque, USA), pp. 2513-2514, 1997.

[8] H. Hermes, Homogeneous coordinates and continuous asymptotically stabilizing feedback controls, vol. 109 of Differential Equations: Stability and Control, pp. 249-260. Marcel Dekker, 1991.

[9] M. Kawski, "Geometric homogeneity and stabilization," in Proc. IFAC Nonlinear Control Symposium (A. Krener and D. Mayne, eds.), (Lake Tahoe, CA), pp. 164-169, 1995.

[10] Y. Orlov, "Finite time stability of homogeneous switched systems," in IEEE Conference on Decision and Control, (Hawaii, USA), pp. 42714276, 2003

[11] S. P. Bhat and D. Bernstein, "Continuous finite-time stabilization of the translational and rotational double integrator," IEEE Trans. Automat. Control, vol. 43, pp. 678-682, may 1998.

[12] T. Floquet, J. P. Barbot, and W. Perruquetti, "Higher-order sliding mode stabilization for a class of nonholonomic perturbed systems," Automatica, vol. 39, no. 6, pp. 1077-1083, 2003.

[13] Y. Hong, "Finite-time stabilization and stabilizability of a class of controllable systems," Systems \& Control Letters, vol. 46, no. 4, pp. 231236, 2002.

[14] E. Moulay and W. Perruquetti, "Finite time stability and stabilization of a class of continuous systems," Journal of Mathematical Analysis and Application, vol. 323, no. 2, pp. 1430-1443, 2006.

[15] C. Qian and W. Lin, "Non-lipschitz continuous stabilizers for nonlinear systems with uncontrollable unstable linearization," Systems \& Control Letters, vol. 16, no. 3, pp. 441-463, 2001.

[16] L. Praly, "Generalized weighted homogeneity and state dependent time scale for linear controllable systems," in Proc. IEEE CDC 97, (San Diego, California USA), pp. 4342-4347, IEEE, 1997.

[17] W. Perruquetti, T. Floquet, and E. Moulay, "Finite-time observers: application to secure communication," IEEE Transactions on Automatic Control, vol. 53, no. 1, pp. 356-360, 2008.

[18] Y. Shen and X. Xia, "Semi-global finite-time observers for nonlinear systems," Automatica, vol. 44, no. 12, pp. 3152-3156, 2008.

[19] T. Menard, E. Moulay, and W. Perruquetti, "A global high-gain finitetime observer," IEEE Transactions on Automatic Control, vol. 55, no. 6, pp. 1500-1506, 2010.

[20] Y. Zhang and Y. Yang, "Finite-time consensus of second-order leaderfollowing multi-agent systems without velocity measurements," Physics Letters A, vol. 377, no. 3-4, pp. 243-249, 2013.

[21] Y. Hong, J. Huang, and Y. Xu, "On an output feedback finite-time stabilization problem," IEEE Trans. Automat. Control, vol. 46, pp. 305$309,2001$.

[22] J. Li and C. Qian, "Global finite-time stabilization by dynamic output feedback for a class of continuous nonlinear systems," IEEE Transactions on Automatic Control, vol. 51, no. 5, pp. 879-884, 2006.

[23] C. Qian and J. Li, "Global output feedback stabilization of uppertriangular nonlinear systems using a homogeneous domination approach," International Journal of Robust and Nonlinear Control, vol. 42, no. 3, pp. $185-200,2006$.

[24] W. Tian, C. Qian, R. Jia, and W. Lin, "State estimation and output feedback stabilization of a class of upper-triangular systems using a homogeneous observer," in Proc. of American Control Conference, (Marriott Waterfront, Baltimore, MD, USA), pp. 4342-4347, 2010.

[25] M. Athans and P. L. Falb, Optimal control : an introduction to the theory and its applications. Lincoln Laboratory publications, McGrawHill, 1966.

[26] E. Ryan, "Singular optimal controls for second-order saturating systems," Internat. J. Control, vol. 3, no. 4, pp. 549-564, 1979. 
[27] W. Wonham, Linear multivariable control : a geometric approach. Springer New York, 3rd ed., 1985.

[28] Y. Orlov, Y. Aoustin, and C. Chevallereau, "Finite time stabilization of a perturbed double integrator - part i: Continuous sliding mode-based output feedback synthesis," IEEE Transactions on Automatic Control, vol. 56, no. 3, pp. 614-618, 2011.

[29] Y. Hong, "H $\mathrm{H}_{\infty}$ control, stabilization, and input-output stability of nonlinear systems with homogeneous properties," Automatica, vol. 37, no. 7, pp. 819-829, 2001.

[30] S. Bhat and D. Bernstein, "Geometric homogeneity with applications to finite-time stability," Mathematics of Control, Signals and Systems, vol. 17, pp. 101-127, 2005.

[31] E. Bernuau, A. Polyakov, D. Efimov, and W. Perruquetti, "ISS and iISS properties of homogeneous systems," in Proc. of European Control Conference, (Zürich, Switzerland), 2013.

[32] E. Bernuau, A. Polyakov, D. Efimov, and W. Perruquetti, "Verification of ISS, iISS and IOSS properties applying weighted homogeneity," Systems \& Control Letters, vol. 62, no. 12, pp. 1159 - 1167, 2013.

[33] E. Bernuau, D. Efimov, W. Perruquetti, and A. Polyakov, "On homogeneity and its application in sliding mode," Journal of the Franklin Institute, vol. 351, no. 4, pp. 1866-1901, 2014.

[34] E. Sontag, "Smooth stabilization implies coprime factorization," IEEE Trans. Automat. Control, vol. 34, no. 4, pp. 435-443, 1989.

[35] Y. Hong, Z. P. Jiang, and G. Feng, "Finite-time input-to-state stability and applications to finite-time control design," SIAM Journal on Control and Optimization, vol. 48, no. 10, pp. 4395-4418, 2010.

[36] E. Ryan, "Universal stabilization of a class of nonlinear systems with homogeneous vector fields," Systems \& Control Letters, vol. 26, pp. 177184, 1995. 